University of Nebraska - Lincoln

DigitalCommons@University of Nebraska - Lincoln

7-15-2005

\title{
Optical Deflection and Temporal Characterization of an Ultrafast Laser-Produced Electron Beam
}

\author{
Sudeep Banerjee \\ University of Michigan, Ann Arbor, Sudeep.Banerjee@asu.edu \\ Scott Sepke \\ University of Michigan, Ann Arbor \\ Rahul Shah \\ University of Michigan, Ann Arbor \\ Anthony Valenzuela \\ University of Michigan, Ann Arbor \\ Anatoly Maksimchuk \\ University of Michigan, tolya@umich.edu
}

See next page for additional authors

Follow this and additional works at: https://digitalcommons.unl.edu/physicsumstadter

Part of the Physics Commons

Banerjee, Sudeep; Sepke, Scott; Shah, Rahul; Valenzuela, Anthony; Maksimchuk, Anatoly; and Umstadter, Donald P., "Optical Deflection and Temporal Characterization of an Ultrafast Laser-Produced Electron Beam" (2005). Donald Umstadter Publications. 17.

https://digitalcommons.unl.edu/physicsumstadter/17

This Article is brought to you for free and open access by the Research Papers in Physics and Astronomy at DigitalCommons@University of Nebraska - Lincoln. It has been accepted for inclusion in Donald Umstadter Publications by an authorized administrator of DigitalCommons@University of Nebraska - Lincoln. 


\section{Authors}

Sudeep Banerjee, Scott Sepke, Rahul Shah, Anthony Valenzuela, Anatoly Maksimchuk, and Donald P. Umstadter 


\title{
Optical Deflection and Temporal Characterization of an Ultrafast Laser-Produced Electron Beam
}

\author{
Sudeep Banerjee, ${ }^{*}$ Scott Sepke, ${ }^{*}$ Rahul Shah, Anthony Valenzuela, Anatoly Maksimchuk, and Donald Umstadter*,† \\ University of Michigan, Ann Arbor, Michigan 48109, USA \\ (Received 18 October 2004; published 15 July 2005)
}

\begin{abstract}
The interaction of a laser-produced electron beam with an ultraintense laser pulse in free space is studied. We show that the optical pulse with $a_{0}=0.5$ imparts momentum to the electron beam, causing it to deflect along the laser propagation direction. The observed 3-degree angular deflection is found to be independent of polarization and in good agreement with a theoretical model for the interaction of free electrons with a tightly focused Gaussian pulse, but only when longitudinal fields are taken into account. This technique is used to temporally characterize a subpicosecond laser-wakefield-driven electron bunch. Applications to electron-beam conditioning are also discussed.
\end{abstract}

DOI: 10.1103/PhysRevLett.95.035004

PACS numbers: 52.38.Kd, 52.38.Ph

With the advent of lasers capable of producing ultrahigh intensity pulses, it is now possible to study the interaction of matter with light at unprecedented levels of field strength characterized by the dimensionless parameter $a_{0}=e E / m \omega$. When $a_{0} \sim 1$, the electric field of the light pulse is large enough that electrons oscillate at relativistic velocities. One application that has received considerable recent attention is the study of laser-accelerated electron beams, which - on account of their low transverse emittance $[1,2]$ - hold promise as injectors for both conventional accelerators and for all optically driven $\mathrm{x}$-ray sources based on nonlinear Thomson scattering [3-5].

A finite optical pulse imparts net momentum to free electrons. At moderately high fields, this momentum is primarily transverse and arises from the intensity gradient due to tight focusing; an electron initially born in the focus will escape with an energy of a few eV [6]. However, at high intensities, there is significant longitudinal electron motion. This has led to proposals for "vacuum acceleration," whereby free electrons would be injected into an ultrahigh intensity laser pulse to produce $\mathrm{GeV}$ electron beams [7-9]. An experiment by Malka et al. [10] showed that an electron could be accelerated to almost $1 \mathrm{MeV}$ by all-optical techniques. However, the model used to analyze these results [11] employed fields that did not obey Maxwell's equations [12]. A more complete model [13] based on previous theoretical [14] and experimental work [15] shows the crucial role of longitudinal fields for tightly focused, high intensity laser pulses.

Electron acceleration by laser generated plasma wakefields has also been demonstrated to produce energetic electron bunches. They are expected to have a temporal length of the order of the laser pulse duration [16], ranging from $30 \mathrm{fs}$ to $>1 \mathrm{ps}$, depending on the laser system being used. A direct measurement of the temporal profile of the electron bunch would serve to elucidate the underlying mechanism as well as a precise estimate of the x-ray pulse from an all-optical synchrotronlike source. Recently, electro-optic techniques have been developed to measure the duration of picosecond electron bunches [17], whereas optical techniques could achieve a resolution $<30$ fs.

In this Letter, we report results on the interaction of a laser-produced electron beam with an ultraintense laser pulse in a "colliding" geometry, where the two intersect at an angle of $135 \mathrm{deg}$. The light pulse imparts longitudinal momentum to the electron beam, leading to the beam being deflected. To the best of our knowledge, we have shown for the first time that the longitudinal fields that are present at the focus of a high intensity laser pulse modify the trajectory of the electron beam at relativistic intensities. We have also shown that the temporal duration of the electron beam is of the order of a picosecond.

The experiments were performed with a hybrid Ti:Sapphire-Nd:Glass laser system that produced pulses of $400 \mathrm{fs}$ duration at $1.053 \mu \mathrm{m}$ with maximum power delivered at the experimental setup of $5 \mathrm{TW}$. The twoinch diameter laser beam is split at the entrance of the experimental chamber with a dielectric-coated beam splitter, which reflects $80 \%$ (primary beam) and transmits $20 \%$ (secondary beam) of the incident pulse. The two beams are transported and then recombined on a supersonic helium nozzle. A schematic of the experimental setup is shown in Fig. 1. Both beams are focused with off-axis paraboloids leading to a focal spot of $8 \mu \mathrm{m}$ in diameter containing $40 \%$ of the total energy. The focused primary laser pulse interacts with helium from the supersonic nozzle to produce an underdense plasma. The electron density has been interferometrically measured to be $\sim 1 \times 10^{19} \mathrm{~cm}^{-3}$.

Under the conditions of our experiment, helium is completely ionized by the rising edge of the laser pulse. As such, the peak of the pulse interacts with a completely ionized medium and is observed to stay self-focused over the entire $1 \mathrm{~mm}$ length of the gas jet [18]. The plasma channeling is observed by an imaging system that looks at the Thomson scattered laser light at $1.053 \mu \mathrm{m}$. The inset to Fig. 1 depicts the channeling of the two laser pulses 


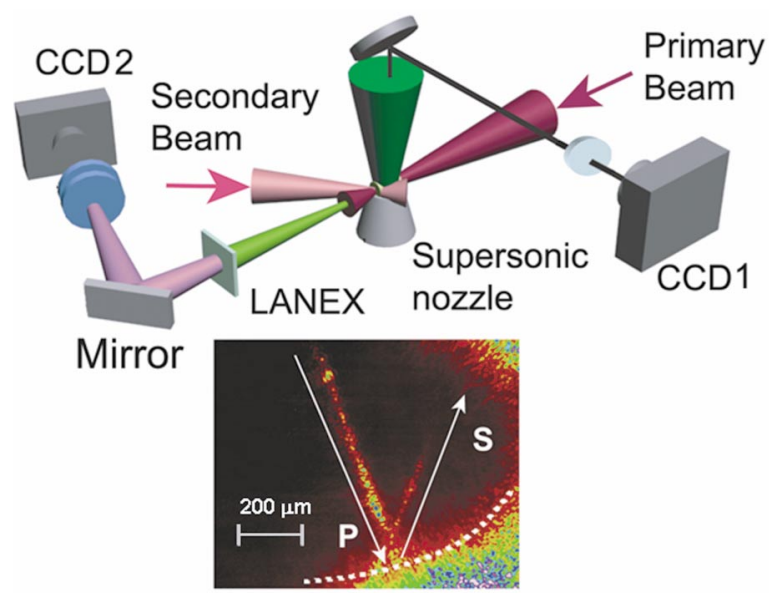

FIG. 1 (color). Schematic of experimental setup to study the deflection of a laser-produced electron beam by a second optical pulse. The dotted line marks the boundary of the nozzle. The primary laser pulse $(P)$ drives the electron beam while the secondary pulse $(S)$ is used for scattering. Shown are the diagnostics for monitoring the channeling of the laser beam (CCD1) and the electron-beam characteristics (CCD2). The inset depicts the channeling of the two pulses through the underdense plasma and shows that the interaction point lies outside of the supersonic flow. The bright region at the intersection point is primarily due to the scattering of the laser beam from the top of the nozzle.

through helium. When the primary laser pulse is focused precisely on the sharp gradient at the entrance to the supersonic nozzle, a well-collimated, energetic electron beam is observed along the direction of laser propagation on a fluorescent screen (LANEX). The secondary laser pulse can be manipulated to ensure precise spatio-temporal overlap with the primary pulse over the entire length of the supersonic nozzle. When the two pulses are overlapped within the plasma, there is significant modification of both the channeling and the electron beam. To avoid such plasma effects, the beams are overlapped at the exit of the nozzle, where the density is $3 \times 10^{18} \mathrm{~cm}^{-3}$, low enough that plasma effects are not significant. The top edge of the supersonic nozzle is $600 \mu \mathrm{m}$ below the vacuum focal spot. The precise spatial location of the two laser pulses is shown in the inset to Fig. 1. The secondary pulse undergoes self-channeling beyond the intersection point, when it experiences higher gas densities.

When the two laser pulses are spatio-temporally overlapped at the exit of the supersonic nozzle, the trajectory of the electron beam driven by the primary laser pulse is modified. Figure 2(a) shows the electron beam produced when only the primary pulse is present. The beam depicted has an angular divergence of $1 \mathrm{deg}$. The energy distribution of the beam is exponential with an effective energy of $520 \mathrm{keV}$ as measured by us and in agreement with previous observations [1]. The angular profile and energy distribution of the beam depend sensitively on the plasma density and laser intensity. The former is fixed by maintaining the

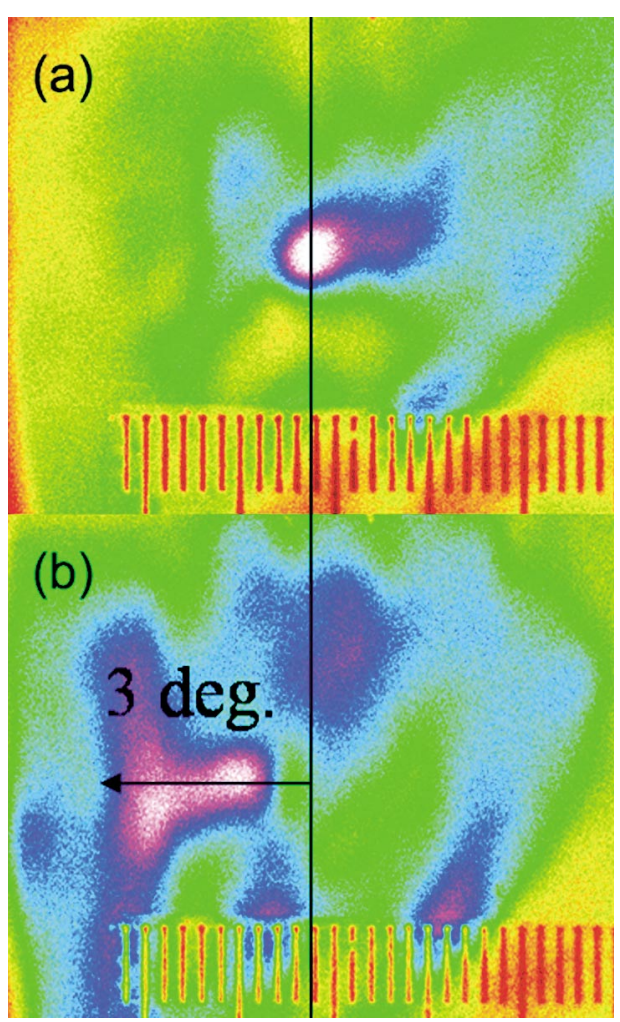

FIG. 2 (color). Spatial profile of the electron beam with (a) one laser pulse and (b) two pulses. The beam deflects in a direction consistent with momentum being imparted to it along the propagation vector of the secondary pulse.

backing pressure on the nozzle at a constant value. For the latter, only those laser shots are considered where the intensity is constant to within $\pm 5 \%$.

Figure 2(b) shows the electron beam when the secondary pulse interacts with the electron beam. The beam becomes elongated, and, based on the experimental configuration, it can be deduced that the electrons are imparted momentum along the direction of propagation of the secondary laser pulse. The transverse gradient of the secondary laser pulse imparts momentum to the beam in the vertical direction and this is also observed. The data shown are for the case of maximum deflection, which is nominally referred to as zero delay. If the two optical pulses are overlapped inside the plasma, a strong enhancement of the Thomson scattered light is observed where the pulses intersect [19], the electron beam degrades in intensity, and does not have a well-defined angular profile. The data clearly show the need for a low divergence beam to achieve the necessary angular resolution. Also, since the effect disappears when the secondary focus is displaced by $5 \mu \mathrm{m}$ vertically with respect to the primary focus, it can be inferred that the electron beam exiting the channel has a transverse spatial extent $<10 \mu \mathrm{m}$.

In order to model the deflection observed above, we have solved the equation of motion $m d(\gamma \mathbf{v}) / d t=-e[\mathbf{E}+$ $\mathbf{v} \times \mathbf{B}]$ for an electron subjected to a linearly polarized, 
focused Gaussian laser pulse with finite temporal extent. An approximate expression for the laser field may be found in terms of a series expansion based on the expansion parameter $\epsilon=1 / k_{0} w_{0}$ where $\mathbf{k}_{0}$ is the wave vector of the laser pulse and $w_{0}$ is the focused spot size. For the current experiment, $\epsilon=0.02$, the first order correction needs to be considered and the nonzero components of the electric field are given by [13].

$$
\begin{gathered}
E_{x}=E_{0} \frac{w_{0}}{w} \exp \left(-\frac{r^{2}}{w^{2}}\right) \sin \left(\Phi_{G}\right) \\
E_{z}=2 E_{0} \epsilon \frac{x w_{0}}{w^{2}} \exp \left(-\frac{r^{2}}{w^{2}}\right) \cos \left(\Phi_{G}^{1}\right) .
\end{gathered}
$$

A similar set of equations can be written down for $B_{y}$ and $B_{z}$. In the above, $\Phi_{G}=\omega_{0} t-k_{0} z+\arctan \left[z / z_{R}\right]-$ $z r^{2} / z_{R} w^{2}-\phi_{0}, \quad \Phi_{G}^{1}=\Phi_{G}+\arctan \left[z / z_{R}\right], \quad w=$ $w_{0} \sqrt{1+z^{2} / z_{R}^{2}}$ is the dependence of the beam waist on longitudinal position, $\phi_{0}$ is an arbitrary constant, and $z_{R}=$ $k_{0} w_{0}^{2} / 2$ is the Rayleigh range. To simulate the electron beam passing through the scattering laser pulse, 121 positions were selected inside of a $10 \mu \mathrm{m}$ square grid, normal to the axis of the primary laser pulse. Electrons were born at each of these points with an initial momentum vector $\mathbf{p}_{0}$ directed 135 degrees relative to the secondary laser axis. Using the laser fields shown above and a cosine squared temporal envelope with a FWHM of $500 \mathrm{fs}$, the fully relativistic equation of motion was then solved for each particle independently using an explicit fifth order RungeKutta solver for each of the 121 initial positions with energies from 75 to $500 \mathrm{keV}$ in $50 \mathrm{keV}$ increments and for various delays. The simulation begins when the leading edge of the pulse intersects the electron and terminates upon the electron exiting the rear of the pulse. The final deflection angle is then defined in radians by $\tan \left(\theta_{D}-\right.$ $\left.\frac{\pi}{4}\right)=\frac{p_{y f}}{p_{z f}}$, where $p_{j f}$ is the final electron momentum in the $j$ th direction. Since the deflection is dependent on the initial electron position, it has been averaged across the cross section of the beam. Figure 3(a) depicts the results of this calculation. The deflection is maximum for low energy electrons and decreases as the electron energy increases.

In order to compare these calculations to the experimental data, the dependence of electron number on both angle and energy needs to be taken into account. We have assumed a uniform energy distribution across the cross section of the beam, an assumption which is reasonable for the lower energy electrons relevant here and has been verified experimentally [1]. The unperturbed angular function is experimentally determined from single beam measurements. Since the deflection depends on the electron energy, an electron at angular position $\theta$ is deflected to a new angle $\theta \rightarrow \theta+\theta_{D}(\gamma)$, where the function $\theta_{D}(\gamma)$ is obtained by calculation and depicted in Fig. 3(a). The
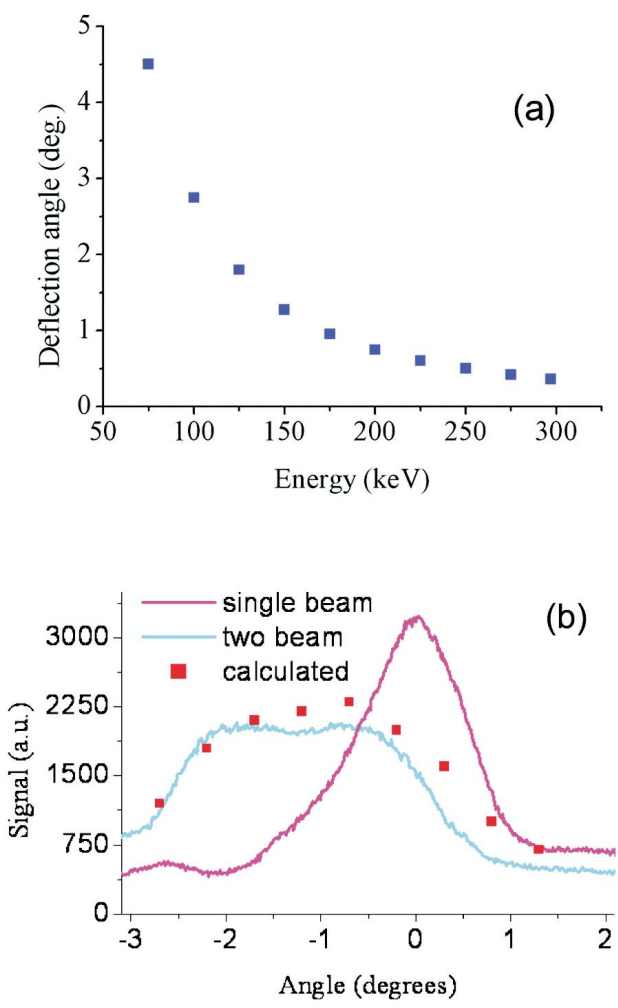

FIG. 3 (color). (a) Calculated mean deflection angle of an electron as a function of its initial energy for $a_{0}=0.5$. (b) Measured and calculated angular profiles of the electron beam with one and two laser pulses.

convolution of angle and energy is used to obtain the angular profile of the electron beam when acted on by the second laser pulse.

The results are depicted in Fig. 3(b). A reasonable agreement is obtained between the experiment and the calculations, validating the claim that the beam is deflected by the ponderomotive force of the secondary laser pulse. We have also performed the above calculations without the longitudinal term. In this case, it is found that the deflection is more than 3 orders of magnitude smaller than that depicted in Fig. 3(a). Interestingly, for the case where the laser and electron beams are copropagating, the electron trajectories with and without longitudinal fields are practically indistinguishable [20].

The polarization independence of these results has been experimentally verified. A half-wave plate was used to rotate the plane of polarization through 90 degrees. The spatial profile of the deflected electron beam was found to be nearly identical for the same delay and laser power, proving that the momentum imparted to the electrons does not depend on the laser polarization. The details of this will be reported elsewhere. We also note that there is insignificant energy change of the electrons, due to the short interaction time between the electron bunch and the laser. This was observed in the experiment by inserting an additional $100 \mu \mathrm{m}$ aluminum filter in front of the fluorescent 


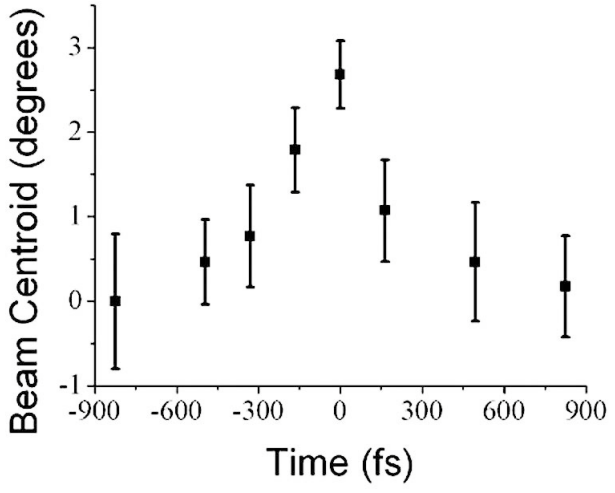

FIG. 4. Evolution of the angular profile of the electron beam as a function of the delay of the secondary pulse. The temporal extent of the deflection is $\sim 1$ ps.

screen. Combined with the filter already mounted on the fluorescent screen this serves to stop electrons with energy $<200 \mathrm{keV}$ and the deflected signal was reduced sixfold.

By varying the delay between the primary and secondary beam, we measured the duration of the electron beam. Figure 4 shows the angular displacement of the beam as a function of time. The mean position of the beam was obtained by the following procedure: the transverse lineout of the spatial profile was averaged along the vertical direction to take into account the spatial dependence of the ponderomotive force. A least-squares fit to a Gaussian profile was then used to obtain the centroid of the beam. The electron beam is deflected for a time spanning about $1 \mathrm{ps}$, which is longer than the duration of the laser pulse but consistent with the temporal profile of the plasma wave previously measured by Le Blanc et al. [21]. This corresponds to the duration of the electron bunch in the energy interval 100-500 keV and could also be used for the measurement of the temporal profile of ultrafast electron beams used for time-resolved x-ray diffraction [22].

In conclusion, we have demonstrated that an energetic, ultrashort, laser-produced electron beam can be deflected by a tightly focused high intensity laser pulse. The deflection depends crucially on the longitudinal fields present at focus, which impart momentum to the electrons along the propagation direction of the laser pulse. The observed deflection is also found to be independent of the laser polarization. The measured temporal duration puts an upper limit on the length of the electron bunch $\sim 1$ ps. Using higher intensity and shorter pulses, this technique can be extended to the measurement of multi-MeV bunch lengths with $<100$ fs resolution. Optical manipulation of the electron trajectories also opens up the possibility of conditioning laser-produced electron beams. Since the magnitude of the deflection depends on the electron energy for a fixed laser intensity, a monochromatic, lowemittance, ultrashort electron beam can be obtained by using a suitable aperture downstream of the interaction region.

The authors gratefully acknowledge support for this work from the National Science Foundation and from the Chemical Sciences, Geosciences and Biosciences Division of the Office of Basic Energy Sciences, U.S. Department of Energy. The laser system and A. M. were supported by the FOCUS Center, University of Michigan. S. S. was supported by Sandia National Laboratories, Department of Defense-Subcontracts, Grant No. 26588.

*Present address: Department of Physics and Astronomy, University of NE, Lincoln, NE 68588.

Corresponding author. Email: dpu@unlserve.unl.edu

[1] S. Y. Chen, A. Maksimchuk, E. Esarey, and D. Umstadter, Phys. Plasmas 6, 4739 (1999).

[2] A. Chao, R. Pitthan, T. Tajima, and D. Yeremian, Phys. Rev. ST Accel. Beams 6, 024201 (2003).

[3] E. S. Sarachik and G. T. Schappert, Phys. Rev. D 1, 2738 (1970).

[4] S.-Y. Chen, A. Maksimchuk, and D. Umstadter, Nature (London) 396, 653 (1998).

[5] S. Banerjee, A.R. Valenzuela, R.C. Shah, A. Maksimchuk, and D. Umstadter, J. Opt. Soc. Am. B 20, 182 (2003); K. Ta Phuoc et al., Phys. Rev. Lett. 91, 195001 (2003).

[6] D. D. Meyerhofer, J. P. Knauer, S. J. McNaught, and C. I. Moore, J. Opt. Soc. Am. B 13, 113 (1996).

[7] J. X. Wang et al., Phys. Rev. E 60, 7473 (1999); S. X. Hu and A. F. Starace, Phys. Rev. Lett. 88, 245003 (2002).

[8] A. Maltsev and T. Ditmire, Phys. Rev. Lett. 90, 053002 (2003).

[9] Y. I. Salamin and C. H. Keitel, Phys. Rev. Lett. 88, 095005 (2002); G. V. Stupakov and M. S. Zolotorev, Phys. Rev. Lett. 86, 5274 (2001).

[10] G. Malka, E. Lefebvre, and J. L. Miquel, Phys. Rev. Lett. 78, 3314 (1997).

[11] F. V. Hartemann et al., Phys. Rev. E 51, 4833 (1995).

[12] P. Mora and B. Quesnel, Phys. Rev. Lett. 80, 1351 (1998).

[13] B. Quesnel and P. Mora, Phys. Rev. E 58, 3719 (1998).

[14] M. Lax, W. H. Louisell, and W. B. Mcknight, Phys. Rev. A 11, 1365 (1975).

[15] B. W. Boreham and H. Hora, Phys. Rev. Lett. 42, 776 (1979).

[16] E. Esarey, C. B. Schroeder, W. P. Leemans, and B. Hafizi, Phys. Plasmas 6, 2262 (1999).

[17] G. Berden et al., Phys. Rev. Lett. 93, 114802 (2004).

[18] G. S. Sarkisov et al., Phys. Rev. E 59, 7042 (1999).

[19] P. Zhang, N. Saleh, S. Chen, Z. M. Sheng, and D. Umstadter, Phys. Rev. Lett. 91, 225001 (2003).

[20] E. Lefebvre, G. Malka, and J. L. Miquel, Phys. Rev. Lett. 80, 1352 (1998).

[21] S. P. Le Blanc et al., Phys. Rev. Lett. 77, 5381 (1996).

[22] B. J. Siwick, J. R. Dwyer, R. E. Jordan, and R. J. D. Miller, Science 302, 1382 (2003). 